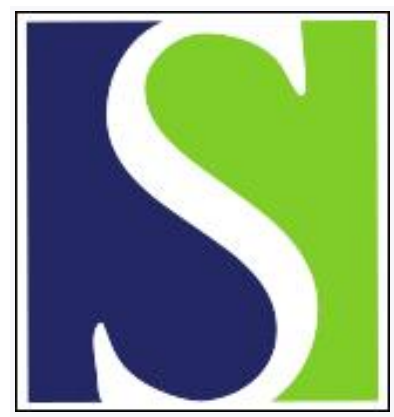

Scand J Work Environ Health 2013;39(6):599-608

https://doi.org/10.5271/sjweh.3368

Published online: 10 May 2013, Issue date: 01 Nov 2013

Does a history of physical exposures at work affect hand-grip strength in midlife? A retrospective cohort study in Denmark by Møller A, Reventlow S, Hansen AMM, Andersen LL, Siersma V, Lund R, Avlund K, Andersen JH, Mortensen OS

This retrospective cohort study evaluates the associations between physical exposures throughout working life and late midlife hand-grip strength (HGS). Exposure to lifting, standing/walking, and kneeling were analyzed in multiple regression models. Physical exposures in working life were associated with a slightly higher HGS among men but no associations between physical exposures and HGS were seen among women.

Affiliation: Department of Occupational Medicine, Køge Sygehus, Lykkebæaekvej 1, DK-4600 Køge, Denmark. annemoller1972@gmail.com

Refers to the following texts of the Journal: 1992;18(2):105-112 2000;26(2):161-168

The following articles refer to this text: 2017;43(5):415-425; 2019;45(6):610-621

Key terms: cohort study; Denmark; ergonomics; hand-grip strength; muscle strength; musculoskeletal aging; occupational; physical activity; physical exposure; retrospective cohort study

This article in PubMed: www.ncbi.nlm.nih.gov/pubmed/23665642

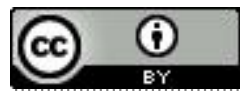




\title{
Does a history of physical exposures at work affect hand-grip strength in midlife? A retrospective cohort study in Denmark
}

\author{
by Anne Møller MD, , 2, 3 Susanne Reventlow DMSc, ${ }^{3}$ Åse Marie Hansen PhD, ${ }^{2,4}$ Lars L Andersen PhD, ${ }^{2}$ \\ Volkert Siersma PhD, ${ }^{3}$ Rikke Lund PhD, ${ }^{4,5}$ Kirsten Avlund DMSc, 4, 5, 6 Johan Hviid Andersen PhD, 7 \\ Ole Steen Mortensen PhD ${ }^{1,2}$
}

\begin{abstract}
Møller A, Reventlow S, Hansen ÅM, Andersen LL, Siersma V, Lund R, Avlund K, Andersen JH, Mortensen OS. Does a history of physical exposures at work affect hand-grip strength in midlife? A retrospective cohort study in Denmark. Scand J Work Environ Health . 2013;39(6):599-608. doi:10.5271/sjweh.3368
\end{abstract}

Objective The aim of this cohort study was to examine associations between physical exposures throughout working life and hand-grip strength (HGS) in midlife.

Methods The Copenhagen Aging and Midlife Biobank (CAMB) provided data about employment and HGS for 3843 Danes. Individual job histories, including duration of employment in specific jobs, were assigned exposures from a job exposure matrix. Exposures were standardized to ton-years (lifting $1000 \mathrm{~kg}$ each day in one year), stand-years (standing/walking for six hours each day in one year) and kneel-years (kneeling for one hour each day in one year). The effects of exposure-years on HGS were analyzed as linear effects and cubic splines in multivariate regression models, adjusted for potential confounders.

Results Mean age was 59 years among both genders and HGS was $49.19 \mathrm{~kg}$ [standard deviation (SD) 8.42] and $30.61 \mathrm{~kg}$ (SD 5.49) among men and women, respectively. Among men, exposure to kneel-years was associated with higher HGS $[>0.030 \mathrm{~kg}(\mathrm{P}=0.007)$ per exposure-year]. Ton- and stand-years were not associated with HGS among either men or women in linear analyses. In spline regression analyses, associations between ton- and stand-years and HGS were non-linear and primarily positive among men. Among women, the associations were non-linear and, according to ton-years, primarily negatively associated with HGS but statistically insignificant.

Conclusion A history of physical exposures at work explained only a minor part of the variation in HGS, though exposure to kneeling throughout working life was associated with a slightly higher HGS among men. Exposure to lifting and standing/walking was not associated with HGS.

Key terms ergonomics; muscle strength; musculoskeletal aging; occupational; physical activity

The influence of work-related exposures on muscle strength has been discussed since the 1980 s, where occupational physical activity was thought to strengthen manual workers (1). Since then the focus has been on the deteriorating effects of physical exposures, which are now known to be important risk factors in the development of musculoskeletal symptoms and diseases $(2,3)$. It has been suggested that exposure and musculoskeletal injury (or deterioration) follow a dose-response relationship (4), but threshold values for duration or intensity of exposure have not been established $(3,5)$. One explanation for this is the multi-factorial origin of musculoskeletal symptoms and diseases, including genetic, morphological, psychological, and biomechanical risk factors (4, 5). Theories about cumulative load suggest "wear and tear" as an important factor in the deterioration of the musculoskeletal system $(4,5)$, and the underlying musculoskeletal aging process also plays an important role (6).

\footnotetext{
${ }^{1}$ Department of Occupational and Environmental Medicine, Køge Sygehus, Køge, Denmark.

2 National Research Centre for the Working Environment, Copenhagen, Denmark.

3 Research Unit for General Practice and Section of General Practice, Department of Public Health, University of Copenhagen, Copenhagen, Denmark.

${ }^{4}$ Section of Social Medicine, Department of Public Health, University of Copenhagen, Copenhagen, Denmark.

5 Center for Healthy Aging, University of Copenhagen, Copenhagen, Denmark.

6 Danish Aging Research Center, Universities of Southern Denmark, Aarhus and Copenhagen, Denmark.

${ }^{7}$ Danish Ramazzini Center, Department of Occupational Medicine, Regional Hospital Herning, Herning, Denmark.
}

Corresponding author: Anne Møller, Department of Occupational Medicine, Køge Sygehus, Lykkebæaekvej 1, DK-4600 Køge, Denmark. [E-mail: annemoller1972@gmail.com] 
Few studies have used the objective outcomes of physical function as signs of occupational musculoskeletal deterioration though objective measures have been suggested to be less biased than self-reports (7). Hand-grip strength (HGS) is a simple objective measure of muscle strength and a well-known predictor of morbidity and disability among older people (8-10) and of mortality among both younger (11) and older age groups $(8,12,13)$. A positive association between manual work and HGS was shown among men with a high workload compared to men with a lower workload in a cross-sectional study (14), suggesting a training effect. A few longitudinal studies have had contradictory results. Savinainen et al (15) found no association between perceived physical workload at baseline and HGS after 22 years of follow-up (mean age 67.3 at follow-up); while Stenholm et al (16) found that self-reports of physical work at baseline were associated with lower HGS at follow-up 22 years later (mean age 48 at follow-up). However, changes in exposure or total amount of exposure during the follow-up period were not taken into account in these studies. Torgen et al (17) included a retrospective assessment of exposures at work and found that HGS was higher among middle-aged manual workers with a history of physical work compared to non-manual workers (17). On the contrary Nygaard et al (18) found no association between current or lifetime physical occupational exposures and HGS among 19-64year old workers.

The inconsistency of results, suggesting both the training and deteriorating effects of physical exposures on midlife HGS, could be due to differences in study design and lack of power in follow-up studies. The aim of this study was to evaluate the association between physical exposures throughout working life and midlife HGS in a large, population-based cohort in Denmark. More specifically, we wanted to study whether a history of physical exposures at work was associated with HGS when we took other determinants of HGS into account. Previous studies of associations between exposures at work and HGS are ambiguous and, based on these, we hypothesized that there would be no association between physical exposures and HGS in midlife.

\section{Methods}

\section{Study design and participants}

This population-based retrospective cohort study included a cross-sectional physical examination as part of the Copenhagen Aging and Midlife Biobank (CAMB) (19). CAMB was established to study signs of early aging among middle-aged Danes and was based on three existing Danish cohorts. In this study, we used data from two of the three cohorts in CAMB: the Metropolit Cohort (MP) and the Danish Longitudinal Study on Work, Unemployment and Health (DALWUH). In total, 12656 middle-aged men and women were invited to take part (see figure 1).

The data collection in CAMB took place between April 2009 and March 2011 and included a postal questionnaire and a health examination at the National Research Centre for the Working Environment (NRCWE). This involved a review of the completed questionnaire, measurement of weight and height, physical tests including measurement of HGS, cognitive tests, blood sampling, and finally information about health status arising from some of the results of the examination. For details about the use of data from CAMB in this study see our research protocols (20). The selection of participants is illustrated in figure 1.

\section{Exposure}

The assessment of physical exposures at work was based on information about job history from the questionnaire combined with data from a job exposure matrix. Selfreports of physical exposures in the workplace derived from the questionnaire were not used since they had low reliability compared with similar information derived from a semi-structured interview (21). The CAMB questionnaire provided job titles and length of service for the participants' five longest-held occupations. Each participant's job history was coded according to the 1988 revision of the Danish version of the International Standard Classification of Occupations register (D-ISCO 88) (20). From an existing Danish job exposure matrix (the knee-hip matrix), information about physical exposures in Danish jobs (linked to D-ISCO-88 codes) was retrieved (22). The knee-hip matrix was based on expert judgments of physical exposures associated with risk of osteoarthritis in the lower limb: sitting, standing/walking, whole-body vibration, kneeling, and lifting (weight and number of heavy lifts) (20). In the present study, we used three physical exposures as proxy measures for occupational physical activity: (i) lifting, because lifting at work is the main physical exposure included in the definition of hard physical work (23); (ii) standing/walking, because standing/walking at work is a common exposure, even in jobs that do not include lifting but are still categorized as physical work, like cleaning; and (iii) kneeling, because kneeling is a specific exposure in physically demanding job types, like floor laying or plumbing.

The total amount of exposure for a study participant was expressed as the number of years a standard daily exposure incurred. Thus, the years of employment in each of the jobs retrieved from the questionnaire were multiplied by the corresponding daily amount of lifting, standing/walking, and kneeling retrieved from the 


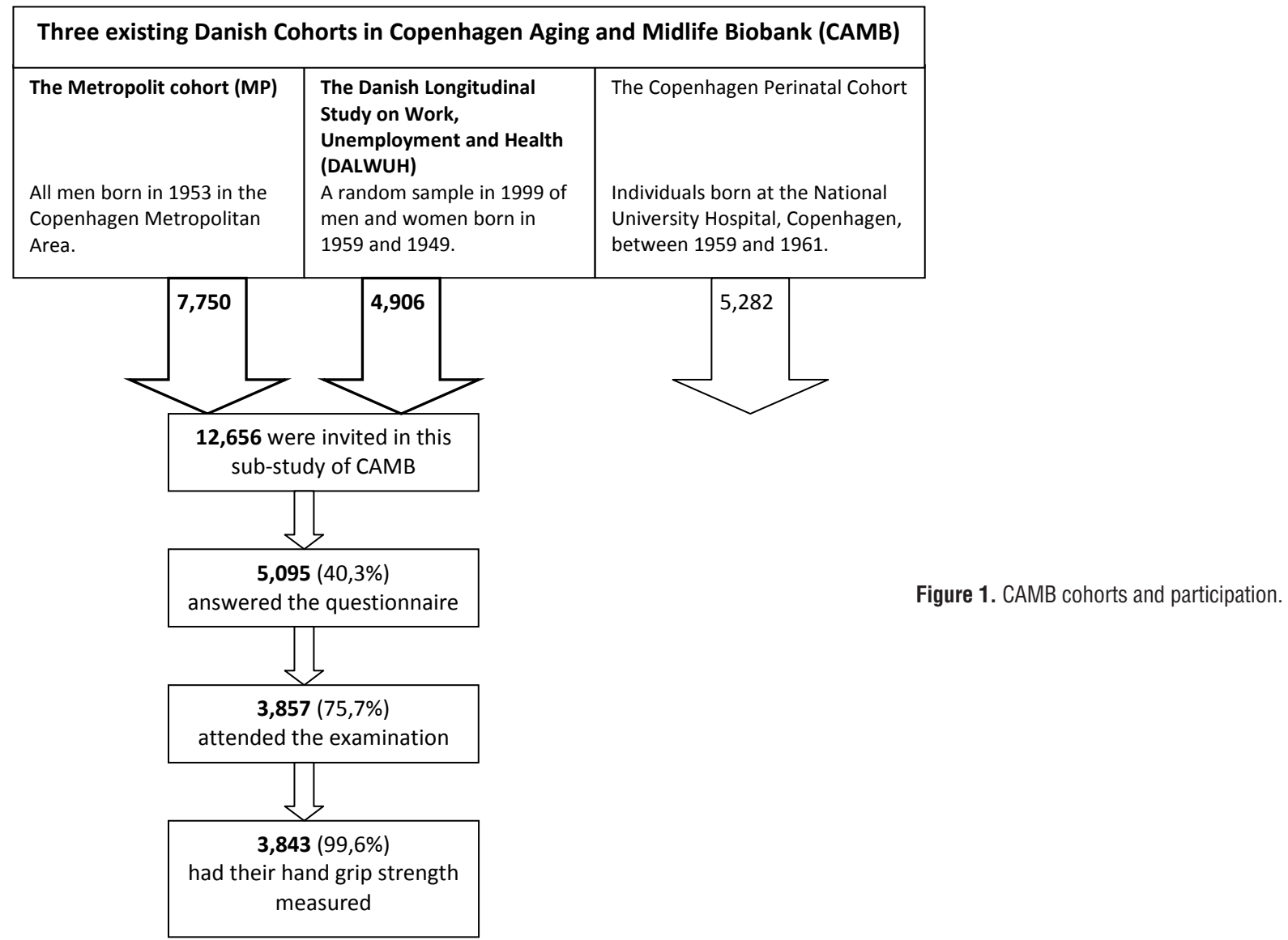

knee-hip matrix, and then calculated for the participants' entire working life. In this way the exposures were standardized as ton-years (lifting $1000 \mathrm{~kg}$ each day in one year), stand-years (standing/walking at work for six hours each day in one year) and kneel-years (kneeling at work for one hour each day in one year).

\section{Outcome}

HGS was measured with a Jamar dynamometer (model G100, Biometrics Ltd, Newport, UK) wired to a computer's signal conditioning interface, enabling automatic recording of the grip strength force. Each participant sat upright in a chair with the elbow flexed at $90^{\circ}$ and was instructed to squeeze the dynamometer as fast and as forcefully as possible (24). The maximum force value $(\mathrm{kg})$ of five possible attempts was defined as the HGS.

\section{Covariates and intermediate variables}

From the CAMB questionnaire, we had information about age, height, weight, vocational education, chronic diseases, leisure-time physical activity (LTPA), and pain in hands and wrists.
Our theoretical model is seen in figure 2. Muscle strength declines over time, and age is an important confounder since it also influences length of exposure. Height and weight (among men) are strongly correlated to HGS (25) and therefore included in the models. Men were included from the MP and DALWUH cohorts therefore "cohort" was included as a covariate. Vocational education is associated with both exposure and outcome and therefore included as a confounder in the theoretical model. Vocational education was categorized into five groups: unskilled, skilled manual worker, and short-, medium-, or long-cycle further education. Chronic diseases influence exposure but could be a result of exposures, too, and therefore a potential mediator in the theoretical model. Number of chronic diseases was registered and grouped in three: 0,1 , and $\geq 2$ chronic diseases. Diseases supposed to influence both exposure and outcome were asthma, diabetes, hypertension, angina pectoris, stroke, myocardial infarction, bronchitis, emphysema, rheumatoid arthritis, osteoarthritis, cancer, anxiety, depression/other psychiatric diseases, and back pain. LTPA influences physical capacity and thereby both exposure and outcome, however the level of physical activity at work likewise influences LTPA. 


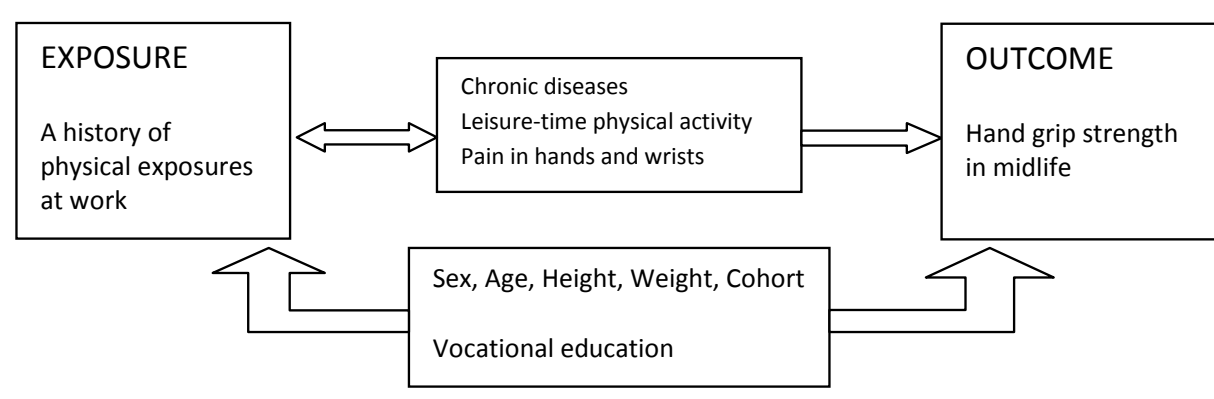

Figure 2. Theoretical model. Associations between exposure and outcome including covariates.
Information about the intensity of weekly physical activity during leisure-time was categorized as medium/ hard $=>4$ hours a week, light $=<4$ hours a week, and sedentary=reading/watching television during leisure time. Pain was a potential mediator too, and pain in one or both hands and wrists was registered on a scale from 1 (no pain) to 9 (worst possible pain).

\section{Statistical methods}

Sex influences both exposures and muscle strength and all analyses were stratified by gender. The associations between exposure-years and HGS, adjusted for potential confounding, were estimated in multivariate linear regression models. Model 1 included age, height, and weight; model 2 included covariates from model 1 and vocational education. Chronic diseases, LTPA and pain in hands/wrists could both be mediators and confounders and thus were introduced separately to model 2 to study the effect on the associations. To evaluate how well the models predict HGS, we report the proportion of the variation explained by the regression models $\left(\mathrm{R}^{2}\right)(26)$. We analyzed for interaction between height and weight and between exposure-years and LTPA. Since the effect of physical exposures on HGS was hypothesized to be a combination of training, deterioration, and aging effects, a linear term may be too limited to characterize this association. Therefore we studied the shape of the association by modeling it as a restricted cubic spline function. The resulting spline functions were then plotted to show the expected increase in HGS attributed to each category of exposure, avoiding a linearity assumption $(27,28)$.

All analyses were performed using SAS 9.2 (SAS Institute, Cary, NC, USA), except the regression with spline functions which were done in the R system for statistical computation.

\section{Results}

The characteristics of the study population are presented in table 1. Mean age was 59 (53-64) years and men constituted $79.2 \%$ of the study population since the MP cohort included only male participants. Mean seniority in work based on the five longest-held employments registered in the CAMB questionnaire was 31.67 (SD 7.85) years among men and 30.10 (SD 8.82) years among women. Mean HGS was 1.6 times higher among men compared to women, and women had fewer exposureyears than men.

In the unadjusted analysis of the association between ton-years and HGS, a significant decline of $0.014 \mathrm{~kg}$ per ton-year was seen among men $(\mathrm{P}=0.045)$. However, when adjusting for age, height, and weight, the association between ton-years and HGS disappeared. In women no significant association was seen in either of the models (table 2). In all unadjusted analyses, the physical exposures explained less than $1 \%$ of the variation in HGS however the full model explained up to $24 \%$ of the variation.

Among men, exposure to kneel-years was positively associated with higher HGS in model 2, increasing HGS by $0.030 \mathrm{~kg}$ per exposure-year $(\mathrm{P}=0.007)$, see table 2 .

In unadjusted analyses of associations between LTPA and HGS, we found that medium/high compared to sedentary LTPA increased HGS by 2.57 and $2.04 \mathrm{~kg}$ among women and men respectively $(\mathrm{P}=0.0025$ and 0.0002$)$. However, when LTPA was introduced to model 2, the association between kneel-years and HGS in men was attenuated only slightly. Having $\geq 2$ chronic diseases decreased HGS by 1.29 and $1.28 \mathrm{~kg}$ among women and men respectively $(\mathrm{P}=0.0059$ and $\mathrm{P}=0.0007)$ in unadjusted analyses. However, when included in model 2, chronic diseases affected the associations only slightly.

There was no significant interaction between height and weight, and only one statistically significant interaction term between ton-years and LTPA among men. Introducing the interaction term to model 2 attenuated the association between ton-years and HGS (from $\beta=-0.003 \mathrm{~kg}, \mathrm{P}=0.653$ to $\beta=0.0003 \mathrm{~kg}, \mathrm{P}=0.9567$ ).

The associations between each of the three physical exposures and HGS were evaluated visually (figure 3). Exposure to ton- and stand-years was slightly positively associated with HGS among men (figure 3, left column). The non-linear regression confirmed the positive asso- 
Table 1. Characteristics of the study population, exposures and outcome. [MP=Metropolit Cohort; DALWUH=Danish Longitudinal Study on Work, Unemployment and Health; SD=standard deviation]

\begin{tabular}{|c|c|c|c|c|c|c|c|c|}
\hline & \multicolumn{4}{|c|}{ Men } & \multicolumn{4}{|c|}{ Women } \\
\hline & $\mathrm{N}$ & $\%$ & Mean & SD & $\mathrm{N}$ & $\%$ & Mean & SD \\
\hline Age & 4035 & & 58.99 & 2.32 & 1060 & & 58.58 & 5.00 \\
\hline Height (cm) & 3968 & & 179.66 & 6.76 & 1045 & & 166.58 & 6.15 \\
\hline Weight (kg) & 3941 & & 85.86 & 14.34 & 1029 & & 69.79 & 13.26 \\
\hline Pain index a & 3964 & & 1.88 & 1.72 & 1044 & & 2.44 & 2.20 \\
\hline Duration of working years ${ }^{b}$ & 3880 & & 31.46 & 8.12 & 1016 & & 29.69 & 8.94 \\
\hline Chronic diseases ${ }^{\mathrm{C}}$ & 3993 & & & & 1052 & & & \\
\hline No disease & 1225 & 30.68 & & & 320 & 30.42 & & \\
\hline 1 disease & 1326 & 33.21 & & & 311 & 29.56 & & \\
\hline$\geq 2$ diseases & 1442 & 36.11 & & & 421 & 40.02 & & \\
\hline Vocational education & 3964 & & & & 1039 & & & \\
\hline Long cycle & 738 & 18.6 & & & 131 & 12.6 & & \\
\hline Medium cycle & 857 & 21.6 & & & 313 & 30.1 & & \\
\hline Short cycle & 336 & 8.5 & & & 107 & 10.3 & & \\
\hline Semi-skilled & 1689 & 42.6 & & & 387 & 37.2 & & \\
\hline Unskilled & 344 & 8.7 & & & 101 & 9.7 & & \\
\hline Intensity of leisure-time physical activity d & 3957 & & & & 1040 & & & \\
\hline Medium/hard & 1253 & 31.7 & & & 255 & 24.5 & & \\
\hline Light & 2240 & 56.6 & & & 706 & 67.9 & & \\
\hline Sedentary & 464 & 11.7 & & & 79 & 7.6 & & \\
\hline Labor market status & 3953 & & & & 1033 & & & \\
\hline Employed & 3561 & 88.3 & & & 802 & 77.6 & & \\
\hline Unemployed e & 474 & 11.7 & & & 231 & 22.4 & & \\
\hline Cohort $^{\dagger}$ & 4035 & & & & 1060 & & & \\
\hline MP & 3153 & 78.1 & & & & & & \\
\hline DALWUH & 882 & 21.9 & & & 1060 & 100.0 & & \\
\hline Ton-years ${ }^{9}$ & 3880 & & 12.90 & 23.15 & 1016 & & 6.04 & 12.38 \\
\hline Stand-years ${ }^{\mathrm{h}}$ & 3880 & & 11.26 & 13.80 & 1016 & & 7.43 & 11.44 \\
\hline Kneel-years i & 3880 & & 7.29 & 14.99 & 1016 & & 1.14 & 2.99 \\
\hline Hand grip strength (kg) & 3059 & & 49.19 & 8.42 & 784 & & 30.61 & 5.49 \\
\hline 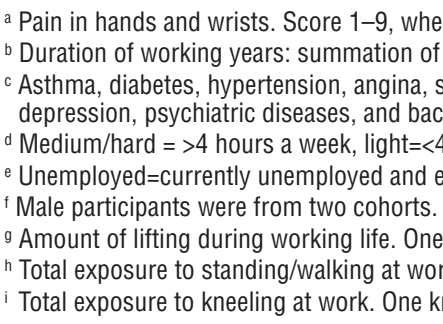 & $\begin{array}{l}1=\text { no pai } \\
\text { ars in the } \\
\text { ke, bron } \\
\text { isease. } \\
\text { urs a w } \\
y \text { retirem } \\
\text { n-year is } \\
\text { One stan } \\
\text { l-year is }\end{array}$ & $\begin{array}{l}\text { nd } 9=\text { wo } \\
\text { e longes } \\
\text { tis, chro } \\
\text {, sedent } \\
\text {, disabil } \\
\text { ing } 100 \\
\text { ear is st }\end{array}$ & $\begin{array}{l}\text { ossible p } \\
\text { iployment } \\
\text { bstructive } \\
\text { reading/w } \\
\text { ensioners } \\
\text { each day } \\
\text { ng/walkin }\end{array}$ & $\begin{array}{l}\text { istered in } \\
\text { nonary di } \\
\text { ng televis }\end{array}$ & $\begin{array}{l}\text { onnaire } \\
\text { Imatoid } \\
\text { leisure- }\end{array}$ & hritis, os & rthritis, ca & r, anxiety \\
\hline
\end{tabular}

ciations found in the linear models according to kneelyears among men, but the association was non-linear. Among women, the associations were non-linear and primarily negative, but all regressions were statistically insignificant (figure 3, right column).

\section{Attrition analyses}

Attrition analyses on the total CAMB study sample, including all three cohorts, showed that responders to the questionnaire (7191/17 938) and participants attending the physical examination (5576/17 938) had significantly higher education and were more likely to be employed compared to non-responders/non-participants (based on data from Danish registers). Use of the healthcare system (ie, visits to the general practitioner during 2009) showed no statistically significant difference among the responders/participants and non-responders/ non-participants, suggesting that participants and nonparticipants did not differ with regard to general health (19). Furthermore, using data from the two cohorts in the present study, we compared those who only completed the questionnaire (1238/5095) with those who took part in both the questionnaire study and the physical tests $(3857 / 5095)$. We found that participants taking the physical tests were exposed to fewer physical exposures at work (11.7 versus 16.9 ton-years among men and 5.1 versus 9.0 ton-years among women), reflecting participants' higher educational attainment level. 
Table 2. Multivariate linear regression models. Associations between exposure-years and hand-grip strength.

\begin{tabular}{|c|c|c|c|c|c|c|c|c|}
\hline & \multicolumn{4}{|c|}{ Men } & \multicolumn{4}{|c|}{ Women } \\
\hline & $\mathrm{N}$ & $\begin{array}{l}\text { Regression } \\
\text { coefficient }\end{array}$ & P-value & $\begin{array}{l}\mathrm{R}^{2 \mathrm{a}} \\
(\%)\end{array}$ & $\mathrm{N}$ & $\begin{array}{l}\text { Regression } \\
\text { coefficient }\end{array}$ & P-value & $\begin{array}{l}\mathrm{R}^{2 \mathrm{a}} \\
(\%)\end{array}$ \\
\hline \multicolumn{9}{|l|}{ Ton-years ${ }^{b}$} \\
\hline Unadjusted & 2986 & -0.014 & 0.045 & 0.13 & 767 & -0.016 & 0.317 & 0.13 \\
\hline Model $1^{c}$ & 2945 & -0.007 & 0.296 & 12.1 & 755 & -0.011 & 0.454 & 21.9 \\
\hline Model 2 d & 2911 & -0.003 & 0.722 & 12.6 & 748 & -0.004 & 0.812 & 23.2 \\
\hline Model 2 and leisure-time physical activity e & 2896 & -0.003 & 0.653 & 13.4 & 745 & -0.001 & 0.928 & 24.9 \\
\hline Model 2 and chronic diseases ${ }^{\dagger}$ & 2909 & -0.001 & 0.864 & 13.0 & 748 & -0.005 & 0.752 & 23.7 \\
\hline Model 2 and pain index ${ }^{g}$ & 2904 & 0.003 & 0.730 & 13.9 & 747 & -0.004 & 0.803 & 24.4 \\
\hline \multicolumn{9}{|l|}{ Stand-years ${ }^{h}$} \\
\hline Unadjusted & 2986 & -0.014 & 0.233 & 0.05 & 767 & -0.008 & 0.667 & 0.02 \\
\hline Model $1^{c}$ & 2945 & 0.010 & 0.339 & 12.1 & 755 & -0.011 & 0.496 & 21.9 \\
\hline Model $2^{d}$ & 2911 & 0.022 & 0.087 & 12.6 & 748 & 0.001 & 0.932 & 23.1 \\
\hline Model 2 and leisure-time physical activity e & 2896 & 0.020 & 0.119 & 13.4 & 745 & 0.002 & 0.908 & 24.9 \\
\hline Model 2 and chronic diseases ${ }^{\dagger}$ & 2909 & 0.024 & 0.063 & 13.1 & 748 & 0.001 & 0.955 & 23.7 \\
\hline Model 2 and pain index $g$ & 2904 & 0.030 & 0.021 & 14.0 & 747 & 0.001 & 0.936 & 24.4 \\
\hline \multicolumn{9}{|l|}{ Kneel-years i } \\
\hline Unadjusted & 2986 & 0.007 & 0.522 & 0.01 & 767 & -0.053 & 0.453 & 0.07 \\
\hline Model $1^{c}$ & 2945 & 0.024 & 0.017 & 12.3 & 755 & -0.070 & 0.271 & 22.0 \\
\hline Model $2^{d}$ & 2911 & 0.030 & 0.007 & 12.8 & 748 & -0.025 & 0.696 & 23.2 \\
\hline Model 2 and leisure-time physical activity e & 2896 & 0.026 & 0.021 & 13.5 & 745 & -0.017 & 0.790 & 24.9 \\
\hline Model 2 and chronic diseases ${ }^{\dagger}$ & 2909 & 0.031 & 0.005 & 13.3 & 748 & -0.030 & 0.645 & 23.8 \\
\hline Model 2 and pain index ${ }^{g}$ & 2904 & 0.036 & 0.001 & 14.2 & 747 & -0.030 & 0.641 & 24.4 \\
\hline
\end{tabular}

a The proportion of the variation explained by the regression model in $\%$.

${ }^{b}$ Amount of lifting during working life. One ton-year is lifting $1000 \mathrm{~kg}$ each day in one year.

${ }^{c}$ Adjusted for age, height, weight, and cohort.

d Adjusted for Model 1 + vocational education.

e Medium/hard=>4 hours a week, light $=<4$ hours a week, sedentary=reading/watching television during leisure-time.

${ }^{f}$ Chronic diseases in three groups: 0,1 or $\geq 2$ of the following diseases: asthma, diabetes, hypertension, angina, stroke, bronchitis, chronic obstructive pulmonary disease, rheumatoid arthritis, osteoarthritis, cancer, anxiety, depression, psychiatric diseases, and back disease.

${ }^{9}$ Pain in hands and wrists. Score 1-9, where 1=no pain and $9=$ worst possible pain.

${ }^{n}$ Total exposure to standing/walking at work. One stand-year is standing/walking for six hours each day in one year.

i Total exposure to kneeling at work. One kneel-year is kneeling at work one hour each day in one year.

\section{Discussion}

A history of physical exposures throughout working life explained only a minor part of the variation in HGS observed in this cohort. A positive association between exposures to kneeling and midlife HGS was seen among men, whereas exposure to lifting and standing/walking was not associated with HGS among men or women.

The effect of exposure to kneel-years in men was non-linear and most pronounced for exposure to 20-40 kneel-years (figure 3). Since the effects on HGS were different for the three outcome measures, one can speculate how jobs including lifting and standing/walking differ from jobs including kneeling according to exposures to the upper limb. It is well known that strong hand movements in vigorous activities increase HGS (25), and maybe the use of hand-held tools in workers kneeling at work (eg, carpenters, floor layers and plumbers) could explain part of the results. In fact, studies have shown that older power line technicians had higher HGS than expected for their age (29) and older waste collectors had higher shoulder strength compared to their younger colleagues (30), suggesting a task-specific training among older workers. Schibye et al (31) compared their results to studies of meat cutters who had lower HGS than waste collectors, maybe due to the lack of variety in job tasks among meat cutters compared to waste collectors (31). Workers with repetitive jobs are standing most of the day, and the jobs might include lifting, but seldom kneeling and thereby earning primarily "stand-years". In our study, an expert judgment of repetitive work was not available from the job exposure matrix, but we found no association between self-reports of repetitive work and HGS in additional analyses (results not shown).

We found no association between physical exposures and HGS among women. This is in accordance with Rantanen et al (32) who found that a history of physical exposures had no association with muscle strength (including HGS) among older women (32). However in spline regressions, we found signs of deteriorative effects of ton-years on HGS among women. This is in contrast to the findings by Torgen et al (17) who found signs of a strengthening effect from the accumulated amount of physical work on HGS among both women and men. Few women had a history of physical exposures and almost no women were exposed to kneeling at work. There was a gender segregation in jobs in this age- 

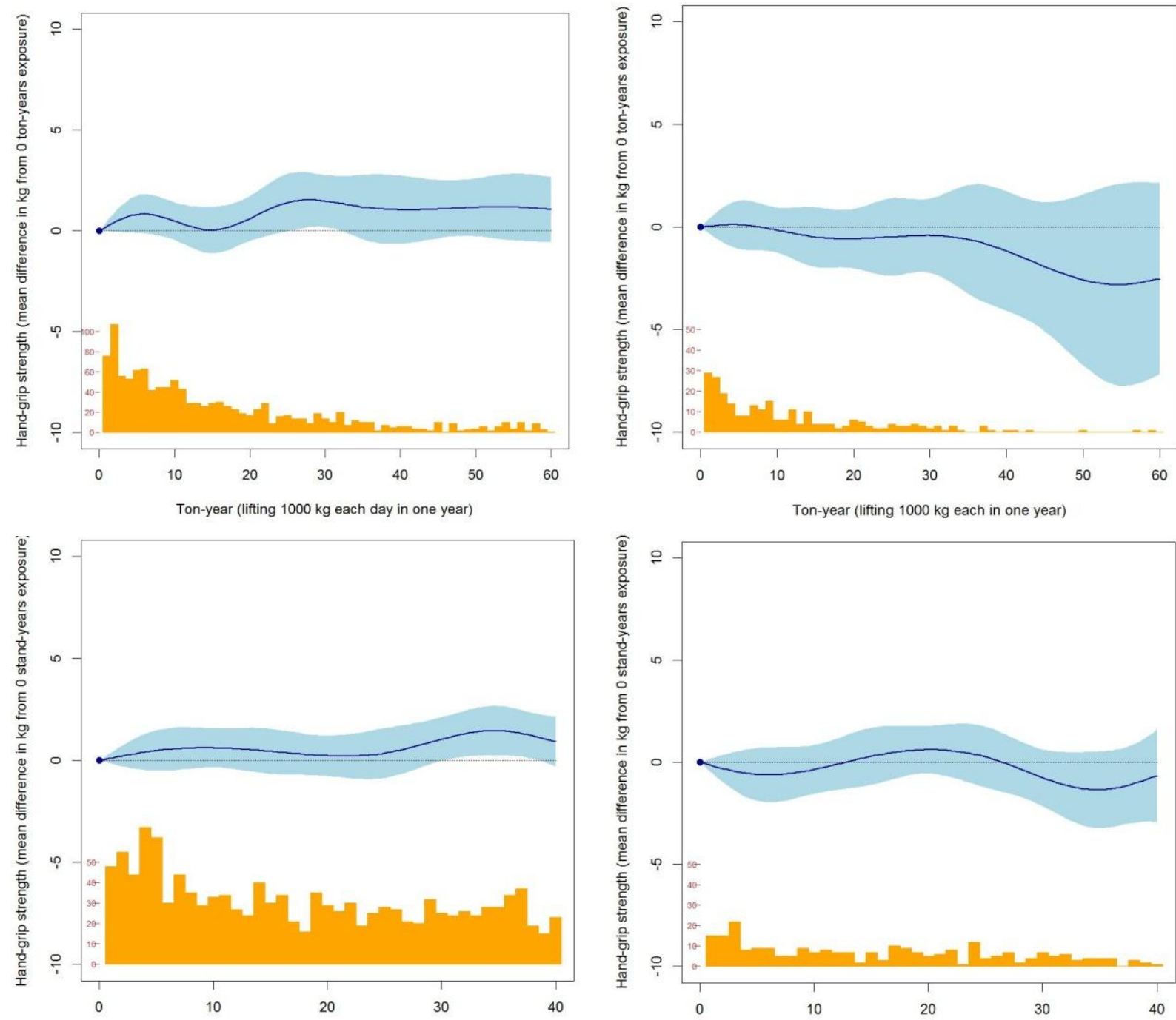

Stand-year (standing/walking at work for six hours each day in one year)

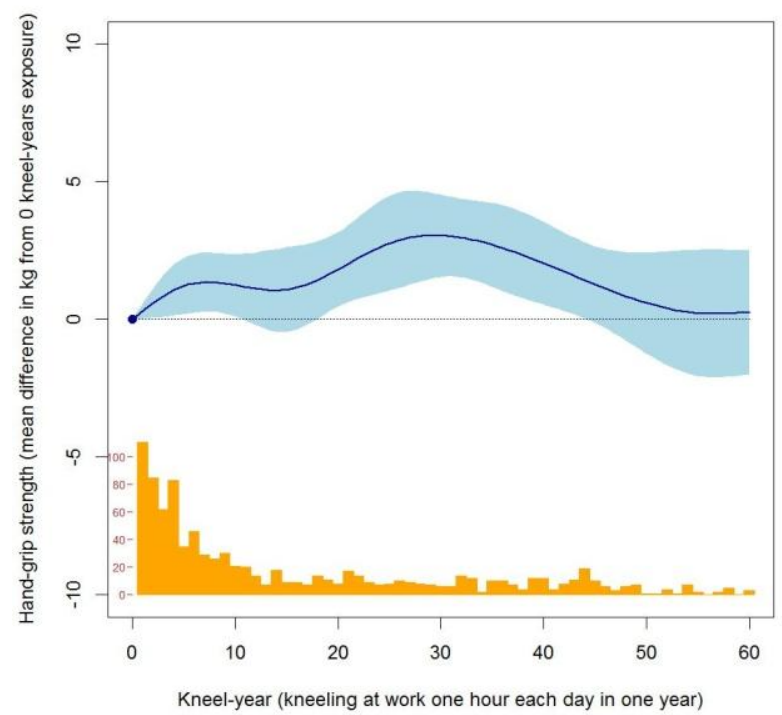

Stand-year (standing/walking at work for six hours each day in one year)

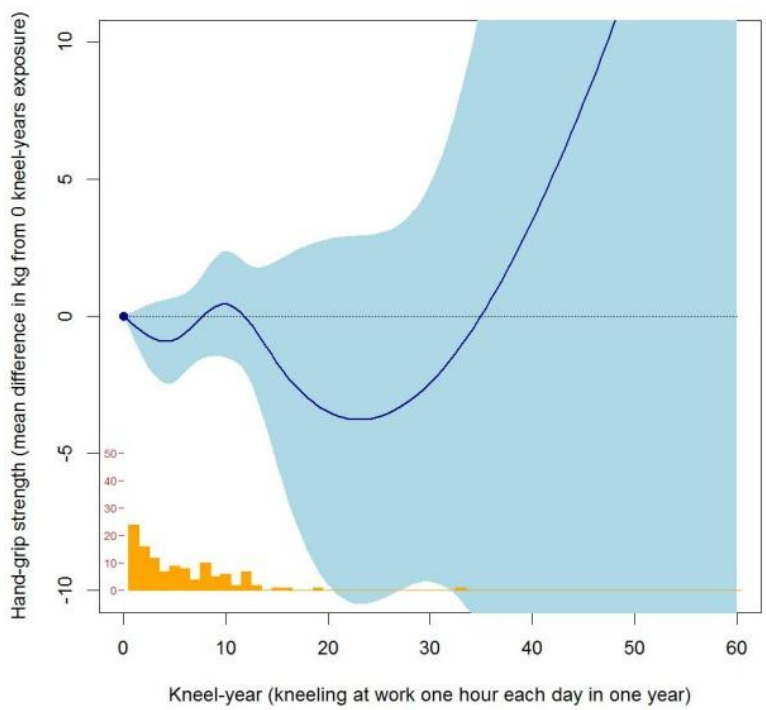

Figure 3. Multivariate spline regression analyses and confidence intervals (model 2 adjusted for height, weigth, age, cohort, and vocational education). Expsoure to ton-, stand-, and kneel-years in years at the x-axis, and hand grip strength (kg) in the y-axis. In men (left column) and women (right column). Participants are visualized in the bottom of each graph. 
cohort since exposed women had mainly been employed as assistant nurses and cleaning assistants, whereas exposed men were employed in many different jobs with a variety of exposures. It is not known whether women would have had the same effect of exposure to kneeling if they had had the same jobs as men. Women have a higher relative workload if they perform the same tasks as men due to their lower muscle strength. Therefore, compared to men, women have another threshold and for example benefit from light physical activity in leisuretime like gardening (33). We found, however, that moderate-to-high activity in leisure-time increased HGS significantly among both men and women in unadjusted analyses. Existing evidence is limited by methodological problems due to categorization and misclassification of physical activity at work and during leisure-time, and also activity related to transportation and household work. Earlier studies have shown ambiguous results: LTPA was beneficial to HGS among middle-aged men (25) whereas no association was seen between baseline physical activity or persistent physical activity and HGS in midlife in a recent follow-up study (16). Interaction between physical exposures at work and physical activity during leisure-time is a possible bias, but we found no interaction between exposures and LTPA except in one exposure group among men. However, inclusion of this interaction term in the regression model did not change the relationship between the exposure and the outcome. Another possible bias, in studies of associations between LTPA and HGS, is participation in sports involving rackets and weight lifting (34). Unfortunately, we had no data on these types of sports in our study.

Introduction of chronic diseases to the models did not change the associations between exposure and outcome. Stenholm et al (16) found that the number of chronic diseases was not associated with HGS but, on the other hand, specific diseases (such as diabetes, hypertension, and asthma) increased the decline in HGS during follow-up. Including a variety of chronic diseases without weighting the diseases or analyzing them separately could be a bias in our study. On the other hand, Torgen et al (17) found that present musculoskeletal symptoms exerted a minor influence on the relationship between physical work and physical capacity. In this study, pain in hands and wrists was associated with lower HGS but explained only an additional $1 \%$ of the variance in HGS among both men and women when included in model 2.

The highest increase in HGS observed in this study (ie, among men with a history of kneeling) was $2 \mathrm{~kg}$; according to our study protocol (20), we regarded a difference of $4 \mathrm{~kg}$ as clinically relevant. Therefore, though the non-linear analyses showed higher HGS among exposed men, the increase in HGS was not as high as expected and not equal among exposure groups. The low correla- tion between physical exposures and HGS in midlife indicates that occupational exposures play only a minor role in the variations of HGS in this age group. In studies that have shown clinically relevant differences in HGS among manual and non-manual workers, results could be biased due to inclusion of both mediators and confounders in the theoretical models [eg, in (35)]. The "healthy worker effect" also plays a role in these studies, since healthy workers remain in the labor market while workers who cannot meet the physical demands of their jobs change occupation or leave the labor market through early retirement or disability pension (29) or, in older cohorts, die during follow-up (36). Our results may also reflect a selection of stronger males taking up physically demanding occupations at an early age (17). We have no information about strength in youth but we do have information about birth weight in one of the CAMB cohorts (the MP cohort). Birth weight is known to be associated with HGS in middle-aged Britons, independently of later height and weight gain (37), however, the associations between physical exposures and HGS were unchanged when we included birth weight in the analyses (results not shown).

Differential drop-out could be another bias in this study since those responding to the questionnaire and attending the physical examination were better educated than non-responders and non-participants. Our attrition analyses showed that non-participants had more exposure-years than participants. It is not known, whether inclusion of the non-responders and non-participants would have changed the associations between exposures and HGS.

\section{Strengths and limitations}

A major strength of this study was the summation of exposures throughout working life based on information from a job exposure matrix. However, job exposure matrices have potential biases too, including the risk of misclassification of exposure (38). Exposures were assigned to job titles in homologous exposure groups, but variation between exposures in the groups could lead to non-differential bias. Changes in exposures over time were another possible bias in this study. Jobs in the 1970s were assigned the same exposures as the same job titles in the 1990s, even though physical exposures at work have declined in the last 40 years (39). However, we expect the effect of this potential bias to be small in this age-homogeneous cohort. Another possible bias is the standardization of exposure. Twenty ton-years can be "earned" in only 10 years of heavy work or 40 years of less heavy work. The physiological effect of these two types of exposure on muscle strength could be different, but is treated equally in our analyses. However, inclusion of seniority at work as a covariate did not change our results (results not shown). 
The large sample size and recruitment from the general population were also strengths of this study and the mean HGS measured in this population corresponds well with recent findings in a Danish population-based cross-sectional study using a Jamar dynamometer (40).

\section{Concluding remarks}

A history of physical exposures at work explained only a minor part of the variation in HGS though exposure to kneeling throughout working life was associated with a slightly higher HGS among men. In future follow-up studies, the age-related decline in HGS in this cohort will be studied also from the perspective of lifetime occupational physical exposures.

\section{References}

1. Nygard C, Luopajarvi T, Suurnakki T, Ilmarinen J. Muscle strength and muscle endurance of middle-aged women and men associated to type, duration and intensity of muscular load at work. Arch Environ Occup Health. 1988;60:291-7. http:// dx.doi.org/10.1007/BF00378476

2. Da Costa B, Vieira E. Risk factors for work-related musculoskeletal disorders: A systematic review of recent longitudinal studies. Am J Ind Med. 2010;53:285-323.

3. Olsen O. Hvidbog om risikofaktorer knyttet til fysisk tungt arbejde. Resume og hovedkonklusioner [White paper on risk factors associated with physically heavy work. Summary and main conclusions]. Copenhagen: The National Research Centre for the Working Environment; 2009. Available from http://www.arbejdsmiljoforskning.dk/da/nyheder/ arkiv/2009/ /media/Praesentationer/risikofaktorer-ogfysisk-tungt-arbejde.pdf

4. Kumar S. Theories of musculoskeletal injury causation. Ergonomics. 2001;44:17-47. http://dx.doi. org/10.1080/00140130120716.

5. Van der Beek A, Frings-Dresen M. Assessment of mechanical exposure in ergonomic epidemiology. Occup Environ Med. 1998;55:291-9. http://dx.doi.org/10.1136/oem.55.5.291

6. Kuh D, Ben-Shlomo Y, Lynch J, Hallqvist J, Power C. Life course epidemiology. J Epidemiol Community Health. 2003;57:778-83. http://dx.doi.org/10.1136/jech.57.10.778.

7. Mohd F, Mackenbach JP, Andersen-Ranberg K, Avendano M. Does socio-economic status predict grip strength in older Europeans? Results from the SHARE study in non-institutionalised men and women aged 50+. J Epidemiol Community Health. 2010;64:829-37. http://dx.doi. org/10.1136/jech.2009.088476.

8. Cesari M, Pahor M, Lauretani F, Zamboni V, Bandinelli $\mathrm{S}$, Bernabei R, et al. Skeletal muscle and mortality results from the InCHIANTI Study. J Gerontol A Biol Sci Med Sci. 2009;64:377-84. http://dx.doi.org/10.1093/gerona/gln031.
9. Cooper R, Kuh D, Cooper C, Gale CR, Lawlor DA, Matthews F, et al. Objective measures of physical capability and subsequent health: a systematic review. Age Ageing. 2011;15:14-23. http://dx.doi.org/10.1093/ageing/afq117.

10. Rantanen T, Guralnik JM, Foley D, Masaki K, Leveille S, Curb JD, et al. Midlife hand grip strength as a predictor of old age disability. JAMA. 1999;281:558-60. http://dx.doi. org/10.1001/jama.281.6.558.

11. Cooper R, Kuh D, Hardy R. Objectively measured physical capability levels and mortality: systematic review and metaanalysis. BMJ. 2010;341:c4467.

12. Avlund K, Osler M, Mortensen EL, Christensen, U Bruunsgaard H, Holm-Pedersen P, et al. Copenhagen Aging and Midlife Biobank (CAMB). An introduction. J Aging Health. 2013 (Accepted for publication).

13. Bohannon RW. Hand-grip dynamometry predicts future outcomes in aging adults. J Geriatr Phys Ther. 2008;31:3-10. http://dx.doi.org/10.1519/00139143-200831010-00002.

14. Gale CR, Martyn CN, Cooper C, Sayer AA. Grip strength, body composition, and mortality. Int J Epidemiol. 2007;36:228-35. http://dx.doi.org/10.1093/ije/dyl224.

15. Ruzic L, Heimer S, Misigoj-Durakovic M, Matkovic B. Increased occupational physical activity does not improve physical fitness. Occup Environ Med. 2003;60:983-5. http:// dx.doi.org/10.1136/oem.60.12.983.

16. Savinainen M, Nygard C, Ilmarinen J. A 16-year follow-up study of physical capacity in relation to perceived workload among ageing employees. Ergonomics. 2004;47:1087-102. http://dx.doi.org/10.1080/00140130410001686357.

17. Stenholm S, Tiainen K, Rantanen T, Sainio P, Heliovaara M, Impivaara $\mathrm{O}$, et al. Long-term determinants of muscle strength decline: prospective evidence from the 22-year mini-Finland follow-up survey. J Am Geriatr Soc. 2012;60:77-85. http:// dx.doi.org/10.1111/j.1532-5415.2011.03779.x.

18. Torgén M, Punnett L, Alfredsson L, Kilbom A. Physical capacity in relation to present and past physical load at work: a study of 484 men and women aged 41 to 58 years. Am J Ind Med. 1999;36:388-400. http://dx.doi. org/10.1002/(SICI)1097-0274(199909)36:3<388::AIDAJIM6>3.0.CO;2-3.

19. Nygård C, Kilbom Å, Wigaeus Hjelm E, Winkel J. Life-time occupational exposure to heavy work and individual physical capacity. Int J Ind Ergon.1994;14:365-72. http://dx.doi. org/10.1016/0169-8141(94)90024-8.

20. Møller A, Mortensen O, Reventlow S, Skov P, Andersen J, Rubak $\mathrm{T}$, et al. Lifetime occupational physical activity and musculoskeletal aging in middle-aged men and women in Denmark: Retrospective cohort study protocol and methods. JMIR Res Protoc. 2012;2:e7. Available from: http://www. researchprotocols.org/2012/2/e7/

21. Møller A, Reventlow S, Andersen JH, Avlund K, Mortensen OS. Validity of Workers' Self-Reports. Evaluation of a Question Assessing Lifetime Exposure to Occupational Physical Activity. Br J Med Med Res. 2012;2:536-52.

22. Rubak TS. Cumulative physical exposure in the work 
environment as a risk factor for primary osteoarthritis leading to total hip replacement. Exposure assessment and risk estimation. Aarhus: Faculty of Health Sciences. Aarhus University; 2010.

23. Mateson L. The functional capacity evaluation. In Anderson G, Demeter S, Smith G, editors. Disability Evaluation. Chicago: Mosby Yearbook; 2003. http://www.epicrehab.com/ abstracts/ama-fce.pdf

24. Fairfax A, Balnave R, Adams R. Variability of grip strength during isometric contraction. Ergonomics.1995;38:1819-30. http://dx.doi.org/10.1080/00140139508925229.

25. Kuh D, Bassey E, Butterworth S, Hardy R, Wadsworth M. Grip strength, postural control, and functional leg power in a representative cohort of British men and women: associations with physical activity, health status, and socioeconomic conditions. J Gerontol A Biol Sci Med Sci. 2005;60:224-31. http://dx.doi.org/10.1093/gerona/60.2.224.

26. Altman DG. Practical Statistics for Medical Research. London: Chapman \& Hall; 1999

27. Steenland K, Deddens J. A practical guide to dose-response analyses and risk assessment in occupational epidemiology. Epidemiology. 2004;15:63-70. http://dx.doi.org/10.1097/01. ede.0000100287.45004.e7.

28. Greenland S. Dose-response and trend analysis in epidemiology: alternatives to categorical analysis. Epidemiology.1995;6:356-65. http://dx.doi.org/10.1097/00001648-199507000-00005.

29. Gall B, Parkhouse W. Changes in physical capacity as a function of age in heavy manual work. Ergonomics. 2004;47:671-87. http://dx.doi.org/10.1080/0014013041000 1658691

30. Schibye B, Hansen A, Søgaard K, Christensen H. Aerobic power and muscle strength among young and elderly workers with and without physically demanding work tasks. Appl Ergon. 2001;32:425-31. http://dx.doi.org/10.1016/S00036870(01)00034-5.

31. Schibye B, Christensen H. The work load during waste collection and meat cutting among workers in different age groups. Arbete och Helsa. 1997;29:272- 278.

32. Rantanen T, Sipilä S, Suominen H, Sipila S. Muscle strength and history of heavy manual work among elderly trained women and randomly chosen sample population. Eur J
Appl Physiol Occup Physiol.1993;66:514-7.http://dx.doi. org/10.1007/BF00634301.

33. Martin HJ, Syddall HE, Dennison EM, Cooper C, Sayer AA. Relationship between customary physical activity, muscle strength and physical performance in older men and women: findings from the Hertfordshire Cohort Study. Age and Ageing. 2008;37:589-93. http://dx.doi.org/10.1093/ageing/afn148.

34. Stamatakis E, Chaudhury M. Temporal trends in adults' sports participation patterns in England between 1997 and 2006: the Health Survey for England. Brit J Sport Med. 2008;42:901-8. http://dx.doi.org/10.1136/bjsm.2008.048082.

35. Russo A, Onder G, Cesari M, Zamboni V, Barillaro C, Capoluongo E, et al. Lifetime occupation and physical function: a prospective cohort study on persons aged 80 years and older living in a community. Occup Environ Med. 2006;63:438-42. http://dx.doi.org/10.1136/oem.2005.023549.

36. Stenholm S, Harkanen T, Sainio P, Heliovaara M, Koskinen S. Long-term Changes in Handgrip Strength in Men and Women-Accounting the Effect of Right Censoring Due to Death. J Gerontol A Biol Sci Med Sci. 2012;67:1068-74. http://dx.doi. org/10.1093/gerona/gls064.

37. Kuh D, Hardy R, Butterworth S, Okell L, Wadsworth M, Cooper $\mathrm{C}$, et al. Developmental origins of midlife grip strength: findings from a birth cohort study. J Gerontol A Biol Sci Med Sci. 2006;61:702-6. http://dx.doi.org/10.1093/ gerona/61.7.702.

38. Kauppinen T, Mutanen PO, Seitsamo J. Magnitude of misclassification bias when using a job-exposure matrix. Scand J Work Environ Health. 1992;18:105-12. http://dx.doi. org/10.5271/sjweh.1604.

39. Torgen M, Kilbom A. Physical work load between 1970 and 1993--did it change? Scand J Work Environ Health. 2000;26:161-8. http://dx.doi.org/10.5271/sjweh.526.

40. Aadahl M, Beyer N, Linneberg A, Thuesen B, Jorgensen T. Grip strength and lower limb extension power in 19-72-yearold Danish men and women: the Health 2006 study. BMJ Open. 2011;1:e00192. http://dx.doi.org/10.1136/ bmjopen-2011-000192.

Received for publication: 8 March 2013 